\title{
A measure-theoretic approach to the invariants of the Selberg class
}

by

J. Kaczorowski (Poznań) and A. Perelli (Genova)

1. Introduction. In [6] and [7] we defined and studied the invariants of the Selberg class $\mathcal{S}$ (to be precise, of the extended Selberg class $\mathcal{S}^{\sharp}$ ). We refer to our survey papers [3], [5], [9] and [10] for the definitions and basic properties of the classes $\mathcal{S}$ and $\mathcal{S}^{\sharp}$. Here we recall that $\mathcal{S}^{\sharp}$ is the class of non-identically vanishing Dirichlet series

$$
F(s)=\sum_{n=1}^{\infty} \frac{a_{n}(F)}{n^{s}}
$$

absolutely convergent for $\sigma>1$, such that $(s-1)^{m} F(s)$ is entire of finite order for some non-negative integer $m$ and $F(s)$ satisfies a functional equation of the form

$$
\Phi(s)=\omega \bar{\Phi}(1-s),
$$

where $\bar{f}(s)=\overline{f(\bar{s})},|\omega|=1$ and

$$
\Phi(s)=Q^{s} \prod_{j=1}^{r} \Gamma\left(\lambda_{j} s+\mu_{j}\right) F(s)=\gamma(s) F(s),
$$

say, with $r \geq 0, Q>0, \lambda_{j}>0$ and $\Re \mu_{j} \geq 0(r=0$ means that there are no $\Gamma$-factors). $\mathcal{S}$ is the subclass of the functions $F \in \mathcal{S}^{\sharp}$ satisfying the Ramanujan conjecture $a_{n}(F) \ll n^{\varepsilon}$ for every $\varepsilon>0$ and having an Euler product of type

$$
\log F(s)=\sum_{n=2}^{\infty} \frac{b_{n}(F)}{n^{s}}
$$

with $b_{n}(F)=0$ unless $n=p^{m}$, and $b_{n}(F) \ll n^{\theta}$ for some $\theta<1 / 2$.

We recall that the notion of invariant of $\mathcal{S}^{\sharp}$ arises from the fact that the data $Q, \lambda_{j}, \mu_{j}$ and $\omega$ of the functional equation of a function $F \in \mathcal{S}^{\sharp}$ are not

Key words and phrases: invariants of the Selberg class, $L$-functions. 
uniquely determined by $F(s)$ (due, essentially, to the multiplication formula for the $\Gamma$ function). Thus, an invariant is an expression defined by means of such data, but depending only on $F(s)$; invariants are denoted by $I$ or by $I_{F}$ or $I(F)$ (particularly when referred to a function $F \in \mathcal{S}^{\sharp}$ ). We refer to [6] and [7] for the meaning of several interesting invariants, such as the degree

$$
d_{F}=2 \sum_{j=1}^{r} \lambda_{j}
$$

the conductor

$$
q_{F}=(2 \pi)^{d_{F}} Q^{2} \prod_{j=1}^{r} \lambda_{j}^{2 \lambda_{j}}
$$

the root number

$$
\omega_{F}^{*}=\omega e^{-i \frac{\pi}{2}\left(\eta_{F}+1\right)}\left(\frac{q_{F}}{(2 \pi)^{d_{F}}}\right)^{i \theta_{F} / d_{F}} \prod_{j=1}^{r} \lambda_{j}^{-2 i \Im \mu_{j}}
$$

and the $H$-invariants

$$
H_{F}(n)=2 \sum_{j=1}^{r} \frac{B_{n}\left(\mu_{j}\right)}{\lambda_{j}^{n-1}}
$$

where $B_{n}(z)$ denotes the $n$th Bernoulli polynomial; for example, $H_{F}(0)=d_{F}$. Note that the root number $\omega_{F}^{*}$ factors as

$$
\omega_{F}^{*}=\left(\omega \prod_{j=1}^{r} \lambda_{j}^{-2 i \Im \mu_{j}}\right)\left(e^{-i \frac{\pi}{2}\left(\eta_{F}+1\right)}\left(\frac{q_{F}}{(2 \pi)^{d_{F}}}\right)^{i \theta_{F} / d_{F}}\right)=\omega_{F}^{\prime} \omega_{F}^{\prime \prime},
$$

say, where $\omega_{F}^{\prime \prime}$ is clearly an invariant, and hence $\omega_{F}^{\prime}$ is an invariant as well. We further recall that an invariant $I$ is called numerical if $I(F) \in \mathbb{C}$ for every $F \in \mathcal{S}^{\sharp}$ (it is easy to construct invariants which are not numerical); in other words, a numerical invariant $I$ is a function $I: \mathcal{S}^{\sharp} \rightarrow \mathbb{C}$. Note that both $\mathcal{S}$ and $\mathcal{S}^{\sharp}$ are multiplicative semigroups, i.e. $F G \in \mathcal{S}$ (resp. $\mathcal{S}^{\sharp}$ ) if $F, G \in \mathcal{S}$ (resp. $\left.\mathcal{S}^{\sharp}\right)$, the $H$-invariants are additive, i.e. $H_{F G}(n)=H_{F}(n)+H_{G}(n)$, and the conductor and $\omega_{F}^{\prime}$ are multiplicative, i.e. $q_{F G}=q_{F} q_{G}$ and $\omega_{F G}^{\prime}=\omega_{F}^{\prime} \omega_{G}^{\prime}$. The set of functions $F \in \mathcal{S}$ (resp. $\left.\mathcal{S}^{\sharp}\right)$ with $d_{F}=d$ is denoted by $\mathcal{S}_{d}\left(\right.$ resp. $\mathcal{S}_{d}^{\sharp}$ ), and the order of the pole of $F(s)$ at $s=1$ is denoted by $m_{F}$.

A fundamental problem in the theory of the Selberg class is describing the admissible values of numerical invariants, i.e. the set of values that such a numerical invariant attains at the functions of $\mathcal{S}$ and $\mathcal{S}^{\sharp}$. For some invariants there are nice conjectures about admissible values, for example the degree conjecture (asserting that $d_{F} \in \mathbb{N}$ for every $F \in \mathcal{S}^{\sharp}$ ) and the conductor conjecture (asserting that $q_{F} \in \mathbb{N}$ for every $F \in \mathcal{S}$ ). In this paper we develop a measure-theoretic approach to this problem. In order to state the results we need some definitions; we will refer to Kechris' book [8] for all the definitions and results needed from topology and measure theory. 
We denote by $\mathbb{R}^{+}$and $\mathbb{C}^{+}$the positive real numbers and the complex numbers with non-negative real part, respectively, and by $T^{1}$ the unit circle. A numerical invariant $I$ is called continuous if for every non-negative integer $r$ there exists a continuous function

$$
f_{I, r}: \mathbb{R}^{+} \times\left(\mathbb{R}^{+} \times \mathbb{C}^{+}\right)^{r} \times T^{1} \rightarrow \mathbb{C}
$$

such that

$$
I(F)=f_{I, r}(Q, \boldsymbol{\lambda}, \boldsymbol{\mu}, \omega)
$$

if $F \in \mathcal{S}^{\sharp}$ satisfies functional equation (1.2), where $\boldsymbol{\lambda}=\left(\lambda_{1}, \ldots, \lambda_{r}\right)$ and $\boldsymbol{\mu}=\left(\mu_{1}, \ldots, \mu_{r}\right)$. Examples of continuous invariants are the $H$-invariants, the conductor and the root numbers $\omega_{F}^{*}, \omega_{F}^{\prime}$ and $\omega_{F}^{\prime \prime}$. Moreover, the real and imaginary parts of a continuous invariant are also continuous invariants.

For technical reasons, it is convenient to work with a slightly more general class than $\mathcal{S}^{\sharp}$, denoted by $\mathcal{S}^{\sharp \sharp}$ and consisting of the Dirichlet series (1.1), absolutely convergent for $\sigma$ sufficiently large and satisfying exactly the same meromorphic continuation and functional equation axioms of $\mathcal{S}^{\sharp}$. Clearly, $\mathcal{S}^{\sharp \sharp}$ is a multiplicative semigroup with identity 1 and $\mathcal{S}, \mathcal{S}^{\sharp}$ are subsemigroups of $\mathcal{S}^{\sharp \sharp}$. Note that the definitions and the main properties pertaining to $\mathcal{S}^{\sharp}$ carry over to $\mathcal{S}^{\sharp \sharp}$. In particular, it is easy to see that Conrey-Ghosh's [1] result that the $\gamma$-factors $\gamma(s)$ of $F(s)$ are uniquely determined up to a constant factor (see also Theorem 8.1 of [5]) holds for $\mathcal{S}^{\sharp \sharp}$ as well, and the invariant theory of $\mathcal{S}^{\sharp}$ carries over to $\mathcal{S}^{\sharp}$.

Let $\mathcal{I}=\left\{I_{j}\right\}_{j \in J}$ with $J \subset \mathbb{N}$ be a countable family of continuous invariants and, for $F, G \in \mathcal{S}^{\sharp}$, write

$$
\varrho_{\mathcal{I}}(F, G)=\sum_{j \in J} \frac{1}{2^{j}} \frac{\left|I_{j}(F)-I_{j}(G)\right|}{1+\left|I_{j}(F)-I_{j}(G)\right|}+\sum_{n=1}^{\infty} \frac{1}{2^{n}} \frac{\left|a_{n}(F)-a_{n}(G)\right|}{1+\left|a_{n}(F)-a_{n}(G)\right|} .
$$

It is easy to check that $\varrho_{\mathcal{I}}$ is a metric on $\mathcal{S}^{\sharp \sharp}$ (recall that if $d(x, y)$ is a metric then so is $d^{\prime}(x, y)=d(x, y) /(1+d(x, y))$, and the proof for $\varrho_{\mathcal{I}}$ is similar $)$. We define the $\mathcal{I}$-Borel sets to be the Borel sets of the metric space $\left(\mathcal{S}^{\sharp \sharp}, \varrho_{\mathcal{I}}\right)$, and we denote by $\mathcal{B}(\mathcal{I})$ the set of the $\mathcal{I}$-Borel sets. We recall that a topological space $X$ is $\sigma$-compact if

$$
X=\bigcup_{n=1}^{\infty} K_{n}
$$

with compact sets $K_{n}$ satisfying $K_{n} \subset K_{n+1}$. Our first result is

TheOREM 1. Let $\mathcal{I}$ be a countable family of continuous invariants. Then $\left(\mathcal{S}^{\sharp \sharp}, \varrho_{\mathcal{I}}\right)$ is a $\sigma$-compact metric space and $\mathcal{S}, \mathcal{S}^{\sharp} \in \mathcal{B}(\mathcal{I})$.

Theorem 1 is a basic topological result from which the following measuretheoretic consequences are deduced. 
TheOREM 2. Let $\mathcal{I}$ be a countable family of continuous invariants. Then $I(B)$ is Lebesgue measurable for every $B \in \mathcal{B}(\mathcal{I})$ and every $I \in \mathcal{I}$.

In particular, from Theorems 1 and 2 we see that $I(\mathcal{S})$ and $I\left(\mathcal{S}^{\sharp}\right)$ are Lebesgue measurable for every continuous invariant $I$. We remark that the measurability of $I(B)$ in Theorem 2 is obtained via Lusin's theorem (see Theorem 21.10 of $[8]$ ), and therefore $I(B)$ is in fact measurable for every $\sigma$-finite Borel measure, although we will only consider the Lebesgue measure in this paper.

$B=\mathcal{S}$ or $B=\mathcal{S}^{\sharp}$ are probably the most interesting cases of Theorem 2, and can be proved by starting directly from a single invariant $I$ (instead of a family $\mathcal{I}$ containing $I$ ); the same remark applies to most cases where a specific set and invariant are involved. However, the definition of the metric by means of a family of invariants allows a convenient and wider choice of Borel sets, and hence a larger range of applications of our results. In fact, for example, adding a continuous invariant $I$ to a family $\mathcal{I}$ we have $\mathcal{B}(\mathcal{I}) \subset \mathcal{B}(\mathcal{I} \cup\{I\})$. As an illustration we state the following simple corollary (examples are given later on).

Corollary 1. Let $I_{0}$ be a continuous invariant and $B \in \mathcal{B}\left(I_{0}\right)$. Then $I(B)$ is Lebesgue measurable for every continuous invariant $I$.

The condition that $B$ is a Borel set in Theorem 2 can be relaxed if we assume more about the invariants of the family $\mathcal{I}$. Given $B \in \mathcal{B}(\mathcal{I})$, let $G$ be the subsemigroup of $\mathcal{S}^{\sharp \sharp}$ generated by $B$; we say that $G$ is an $\mathcal{I}$-Borel generated semigroup.

THEOREM 3. Let $\mathcal{I}$ be a countable family of continuous invariants such that every $I \in \mathcal{I}$ is additive or multiplicative. Then $I(G)$ is Lebesgue measurable for every $\mathcal{I}$-Borel generated semigroup $G$ and every $I \in \mathcal{I}$.

In analogy with Corollary 1 , here is a corollary illustrating the usefulness of the family $\mathcal{I}$.

COROLlary 2. Let $I_{0}$ be an additive or multiplicative continuous invariant, $B \in \mathcal{B}\left(I_{0}\right)$, and $G$ the semigroup generated by $B$. Then $I(G)$ is Lebesgue measurable for every additive or multiplicative continuous invariant $I$.

Of course, the set $B$ in Corollaries 1 and 2 can be intersected with $\mathcal{S}$ or $\mathcal{S}^{\sharp}$, and the conclusions still hold.

Of particular interest are the subsemigroups $G$ of $\mathcal{S}^{\sharp \sharp}$ such that $I(G)$ is Lebesgue measurable for an invariant $I$ (not necessarily continuous). In such a case, $G$ is called an $I$-measurable semigroup. In view of Theorem 3, a first class of examples of such semigroups is given by the $\mathcal{I}$-Borel generated semigroups with all $I \in \mathcal{I}$ additive or multiplicative. Another class of examples (not disjoint from the previous one) is provided by Theorem 2 
and consists of the $\mathcal{I}$-Borel semigroups, that is, the $\mathcal{I}$-Borel sets which are semigroups themselves. Explicit examples of measurable semigroups are as follows. First of all, by Theorem $1, \mathcal{S}$ and $\mathcal{S}^{\sharp}$ are $I$-measurable for every continuous $I$. Other examples of semigroups $I$-measurable for every continuous $I$ are $\mathcal{S}_{0}$ and $\mathcal{S}_{0}^{\sharp}$. In fact, these sets are semigroups and Corollary 1 can clearly be applied. We recall (see [1] and [4]) that $\mathcal{S}_{0}=\{1\}$ and $\mathcal{S}_{0}^{\sharp}$ is a certain set of Dirichlet polynomials. Moreover, thanks to Corollary 2, the following are examples of semigroups $I$-measurable for every additive or multiplicative continuous $I$. Recalling that $d$ denotes the degree, $G^{\text {Dir }}$, generated by $d^{-1}(\{1\}) \cap \mathcal{S}=\mathcal{S}_{1}$, is the semigroup generated by the Riemann zeta function and the shifted Dirichlet $L$-functions (see [4]). $G^{(1)}$, generated by $d^{-1}(\{1\}) \cap \mathcal{S}^{\sharp}=\mathcal{S}_{1}^{\sharp}$, can also be explicitly described (see [4]). Finally, we also mention $G^{(2)}$, generated by $d^{-1}(\{2\}) \cap \mathcal{S}^{\sharp}=\mathcal{S}_{2}^{\sharp}$.

In the case of $I$-measurable semigroups $G$ with $I$ additive or multiplicative we can say more about $\mu(I(G))$, where $\mu$ denotes the Lebesgue measure. Indeed, we have the following simple 0-1 laws for additive and multiplicative invariants.

TheOREM 4. Let $G$ be an I-measurable semigroup. If $I$ is additive and real-valued, then either $\mu(I(G))=0$ or $I(G)$ contains a half-line. If $I$ is multiplicative and takes values in $T^{1}\left(\right.$ resp. $\left.\mathbb{R}^{+}\right)$, then either $\mu(I(G))=0$ or $I(G)=T^{1}($ resp. $I(G)$ contains a half-line $)$.

As is clear from the above discussion, Theorem 4 is closely related to Theorems 2 and 3 . In fact, from Theorems $2-4$ we easily deduce the following consequences. In view of the degree conjecture, the first part of Theorem 4 is particularly interesting in the case of the degree $d$, where $\mu\left(d\left(\mathcal{S}^{\sharp}\right)\right)=0$ is expected. Examples of measurable semigroups $G$ with $\mu(d(G))=0$ are $\mathcal{S}_{0}$, $\mathcal{S}_{0}^{\sharp}, G^{\text {Dir }}$ and $G^{(1)}$.

The most interesting special case of the second part of Theorem 4 is the conductor $q$, and the conductor conjecture suggests that $\mu(q(\mathcal{S}))=0$. For example, it follows from the characterization of the functions of degree 0 and 1 of $\mathcal{S}$ and $\mathcal{S}^{\sharp}$ (see [4]) that

$$
\mu\left(q\left(\mathcal{S}_{0}\right)\right)=\mu\left(q\left(\mathcal{S}_{0}^{\sharp}\right)\right)=\mu\left(q\left(G^{\text {Dir }}\right)\right)=\mu\left(q\left(G^{(1)}\right)\right)=0 .
$$

However, probably $q\left(\mathcal{S}^{\sharp}\right)$ contains a half-line. In fact, in view of Hecke's theory for the groups $G(\lambda)$ (see Hecke's book [2]), already $q\left(G^{(2)}\right)$ will probably contain a half-line.

Another interesting multiplicative invariant is the root number $\omega_{F}^{\prime}$ defined by (1.3). In view of [4] we have $\mu\left(\omega^{\prime}\left(\mathcal{S}_{1}\right)\right)=0$, while $\omega^{\prime}\left(G^{(1)}\right)=T^{1}$. Moreover, since the weight $k$ in Hecke's theory with $\lambda>2$ is arbitrary, it is very likely that $\omega^{\prime}\left(G^{(2)}\right)=T^{1}$. 
We finally remark that in all known or conjectural cases, if the set of values of a continuous invariant has 0 -measure, then it is countable. We therefore state the following conjecture, clearly related to Theorem 4 .

CONJECTURE. Let I be a continuous invariant and $G$ be an I-measurable semigroup. If $I$ is additive or multiplicative with values in $\mathbb{R}^{+}$, then either $I(G)$ is countable or it contains a half-line.

A similar conjecture can be made for multiplicative continuous invariants with values in $T^{1}$; in this case, either $I(G)$ is countable or $I(G)=T^{1}$.

2. Proofs. In order to prove Theorem 1 we need three lemmas.

Lemma 1. Let $\mathcal{I}$ be a countable family of continuous invariants. Then for every $n=1,2, \ldots$ and every $I \in \mathcal{I}$, the functions $F \mapsto a_{n}(F)$ and $F \mapsto I(F)$ are continuous with respect to the metric $\varrho_{\mathcal{I}}$.

Proof. Given a sequence $F_{m} \rightarrow F_{0}$ in $\left(\mathcal{S}^{\sharp \sharp}, \varrho_{\mathcal{I}}\right)$ we have $\varrho_{\mathcal{I}}\left(F_{m}, F_{0}\right) \rightarrow 0$, hence, in particular,

$$
\frac{\left|a_{n}\left(F_{m}\right)-a_{n}\left(F_{0}\right)\right|}{1+\left|a_{n}\left(F_{m}\right)-a_{n}\left(F_{0}\right)\right|} \rightarrow 0 \quad \text { and } \quad \frac{\left|I_{j}\left(F_{m}\right)-I_{j}\left(F_{0}\right)\right|}{1+\left|I_{j}\left(F_{m}\right)-I_{j}\left(F_{0}\right)\right|} \rightarrow 0
$$

whence $a_{n}\left(F_{m}\right) \rightarrow a_{n}\left(F_{0}\right)$ and $I_{j}\left(F_{m}\right) \rightarrow I_{j}\left(F_{0}\right)$.

For $R \geq 2$ integer, let $\mathcal{S}^{\sharp \sharp}(R)$ be the set of $F \in \mathcal{S}^{\sharp \sharp}$ such that

$$
r \leq R, \quad \frac{1}{R} \leq Q, \lambda_{j} \leq R, \quad\left|\mu_{j}\right| \leq R, \quad m_{F} \leq R, \quad \sum_{n=1}^{\infty} \frac{\left|a_{n}(F)\right|}{n^{R}} \leq R
$$

and

$$
|F(s)| \leq e^{|s|^{R}} \quad \text { for }|s| \geq 2 .
$$

Clearly, $\mathcal{S}^{\sharp \sharp}(R) \subset \mathcal{S}^{\sharp \sharp}(R+1)$ and

$$
\mathcal{S}^{\sharp}=\bigcup_{R=2}^{\infty} \mathcal{S}^{\sharp}(R) \text {. }
$$

Lemma 2. Let $\mathcal{I}$ be a countable set of continuous invariants. Then for $R=2,3, \ldots, \mathcal{S}^{\sharp \sharp}(R)$ is a compact subset of $\left(\mathcal{S}^{\sharp \sharp}, \varrho_{\mathcal{I}}\right)$.

Proof. Let $F_{m} \in \mathcal{S}^{\sharp \sharp}(R), m=1,2, \ldots$ By the compactness of closed bounded intervals of $\mathbb{R}$, there exists a subsequence, which for ease of notation we still denote by $\left(F_{m}\right)$, such that $r_{m}=r_{0} \leq R$ and $m_{F_{m}}=m_{0} \leq R$ for every $m$, and the sequences $\left(Q_{m}\right),\left(\lambda_{j, m}\right),\left(\mu_{j, m}\right),\left(\omega_{m}\right)$ and $\left(a_{n}\left(F_{m}\right)\right)$ are convergent to $Q_{0}, \lambda_{j, 0}, \mu_{j, 0}, \omega_{0}$ and $a_{n, 0}$, respectively, all satisfying the above bounds. For $\sigma>R$ we put

$$
F_{0}(s)=\sum_{n=1}^{\infty} \frac{a_{n, 0}}{n^{s}}
$$


which is well defined since as $m \rightarrow \infty$,

$$
\sum_{n=1}^{\infty} \frac{\left|a_{n}\left(F_{m}\right)\right|}{n^{R}} \rightarrow \sum_{n=1}^{\infty} \frac{\left|a_{n, 0}\right|}{n^{R}} \leq R .
$$

Our aim now is to prove that $F_{0} \in \mathcal{S}^{\sharp \sharp}(R)$ and $F_{m}(s) \rightarrow F_{0}(s)$ as $m \rightarrow \infty$, with respect to the metric $\varrho_{\mathcal{I}}$, thus showing that $\mathcal{S}_{\sharp}(R)$ is compact.

We first prove that $F_{0} \in \mathcal{S}^{\sharp \sharp}(R)$. By the definition of $\mathcal{S}^{\sharp \sharp}(R)$ and the choice of $m_{0}$ the functions

$$
H_{m}(s)=(s-1)^{m_{0}} F_{m}(s)
$$

are entire of order $\leq R$. Moreover, by the functional equation, for $t \in \mathbb{R}$ we have

$$
\begin{aligned}
\left|H_{m}(1-R+i t)\right| & \leq(R+|t|)^{R} \frac{\left|\gamma_{F_{m}}(R+i t)\right|}{\left|\gamma_{F_{m}}(1-R+i t)\right|}\left|F_{m}(R+i t)\right| \\
& \leq c_{0}(R)(|t|+2)^{c_{1}(R)}
\end{aligned}
$$

for some constants $c_{j}(R), j=0,1$, hence by the Phragmén-Lindelöf theorem we get

$$
\left|H_{m}(\sigma+i t)\right| \leq c_{0}(R)(|t|+2)^{c_{1}(R)}, \quad \sigma \geq 1-R .
$$

Hence there exists a subsequence of $\left(H_{m}(s)\right)$ which converges to

$$
H_{0}(s)=(s-1)^{m_{0}} \widetilde{F_{0}}(s)
$$

uniformly over compact sets in $\sigma \geq 1-R$; note that $\widetilde{F_{0}}(s)$ is meromorphic for $\sigma>1-R$ with at most a pole of order $\leq R$ at $s=1$. But $\left(H_{m}(s)\right)$ is convergent to $(s-1)^{m_{0}} F_{0}(s)$ for $\sigma>R$, thus $F_{0}(s)=\widetilde{F_{0}}(s)$ for $\sigma>R$, giving a meromorphic continuation of $F_{0}(s)$ to $\sigma \geq 1-R$ with at most a pole of order $\leq R$ at $s=1$. Writing

$$
\gamma_{0}(s)=Q_{0}^{s} \prod_{j=1}^{r_{0}} \Gamma\left(\lambda_{j, 0} s+\mu_{j, 0}\right)
$$

we have

$$
\gamma_{0}(s)=\lim _{m \rightarrow \infty} \gamma_{m}(s), \quad \gamma_{m}(s)=Q_{m}^{s} \prod_{j=1}^{r_{0}} \Gamma\left(\lambda_{j, m} s+\mu_{j, m}\right)
$$

uniformly over compact sets of $\mathbb{C}$ not containing the poles of the $\gamma_{m}(s)$ 's, and for $1-R \leq \sigma \leq R$ the function $F_{0}(s)$ satisfies the functional equation

$$
\gamma_{0}(s) F_{0}(s)=\omega_{0} \bar{\gamma}_{0}(s) \bar{F}_{0}(s) .
$$

This provides a meromorphic continuation of $F_{0}(s)$ to $\mathbb{C}$. Moreover, the bound

$$
\left|F_{0}(s)\right| \leq e^{|s|^{R}}, \quad|s| \geq 2
$$


follows by a limiting process from the same bounds for the $F_{m}(s)$ 's. Thus, $F_{0} \in \mathcal{S}^{\sharp \sharp}(R)$ in view of $(2.2)-(2.4)$.

Finally, since the $I_{j}$ are continuous invariants and $a_{n}\left(F_{m}\right) \rightarrow a_{n}\left(F_{0}\right)$, from (1.4) we have for every positive $\varepsilon$

$$
\begin{aligned}
\varrho_{\mathcal{I}}\left(F_{m}, F_{0}\right)= & \sum_{j \in J} \frac{1}{2^{j}} \frac{\left|I_{j}\left(F_{m}\right)-I_{j}\left(F_{0}\right)\right|}{1+\left|I_{j}\left(F_{m}\right)-I_{j}\left(F_{0}\right)\right|}+\sum_{n=1}^{\infty} \frac{1}{2^{n}} \frac{\left|a_{n}\left(F_{m}\right)-a_{n}\left(F_{0}\right)\right|}{1+\left|a_{n}\left(F_{m}\right)-a_{n}\left(F_{0}\right)\right|} \\
\leq & \sum_{j \in J \cap[1, N]}\left|f_{I_{j}, r_{0}}\left(Q_{m}, \boldsymbol{\lambda}_{m}, \boldsymbol{\mu}_{m}, \omega_{m}\right)-f_{I_{j}, r_{0}}\left(Q_{0}, \boldsymbol{\lambda}_{0}, \boldsymbol{\mu}_{0}, \omega_{0}\right)\right| \\
& +\sum_{j=1}^{N}\left|a_{n}\left(F_{m}\right)-a_{n}\left(F_{0}\right)\right|+\frac{\varepsilon}{3} \leq \varepsilon
\end{aligned}
$$

for $N$ and $m \geq m_{0}(N)$ sufficiently large, so $\varrho_{\mathcal{I}}\left(F_{m}, F_{0}\right) \rightarrow 0$ and the lemma follows.

Lemma 3. Let $\mathcal{I}$ be a countable set of continuous invariants. Then $\mathcal{S}, \mathcal{S}^{\sharp} \in \mathcal{B}(\mathcal{I})$.

Proof. From the well known formula for the abscissa of absolute convergence of Dirichlet series we see that a function $F \in \mathcal{S}^{\sharp \sharp}$ belongs to $\mathcal{S}^{\sharp}$ if and only if for every $\varepsilon>0$ and $N \in \mathbb{N}, N>N(\varepsilon)$,

$$
\sum_{n \leq N}\left|a_{n}(F)\right| \leq N^{1+\varepsilon} .
$$

Therefore, for $\varepsilon>0$ and $N \in \mathbb{N}$ we consider the function $f_{N, \varepsilon}: \mathcal{S}^{\sharp \sharp} \rightarrow \mathbb{R}$ defined by

$$
f_{N, \varepsilon}(F)=\frac{1}{N^{1+\varepsilon}} \sum_{n \leq N}\left|a_{n}(F)\right| .
$$

Since $F \mapsto a_{n}(F)$ is continuous with respect to $\varrho_{\mathcal{I}}, f_{N, \varepsilon}(F)$ is also continuous with respect to $\varrho_{\mathcal{I}}$. Moreover, $\mathcal{S}^{\sharp}$ can be characterized as

$$
\mathcal{S}^{\sharp}=\bigcap_{\varepsilon \in \mathbb{Q}^{+}} \bigcup_{M=1}^{\infty} \bigcap_{N=M}^{\infty} f_{N, \varepsilon}^{-1}([0,1]),
$$

where $\mathbb{Q}^{+}$denotes the positive rational numbers. Since $f_{N, \varepsilon}^{-1}([0,1])$ is a closed subset of $\mathcal{S}^{\sharp \sharp},(2.5)$ shows that $\mathcal{S}^{\sharp}$ is a Borel subset of $\mathcal{S}^{\sharp \sharp}$.

In order to deal with $\mathcal{S}$ we first consider

$$
\mathcal{S}^{\sharp}(1)=\left\{F \in \mathcal{S}^{\sharp}: a_{1}(F)=1\right\}=\mathcal{S}^{\sharp} \cap a_{1}^{-1}(\{1\}) .
$$

In view of the first part of the lemma, $\mathcal{S}^{\sharp}(1)$ is a Borel subset of $\mathcal{S}^{\sharp \sharp}$. For 
$F \in \mathcal{S}^{\sharp}(1)$ let $\sigma_{1}(F) \geq 1$ be such that

$$
\sum_{n=2}^{\infty} \frac{\left|a_{n}(F)\right|}{n^{\sigma}}<1 \quad \text { for } \sigma>\sigma_{1}(F) .
$$

Then for $\sigma>\sigma_{1}(F)$ the function $\log F(s)$ is well defined, and by Taylor's expansion we have

$$
\log F(s)=\sum_{n=2}^{\infty} \frac{b_{n}(F)}{n^{s}}
$$

with

$$
b_{n}(F)=\sum_{m=1}^{\Omega(n)} \frac{(-1)^{m+1}}{m} \sum_{\substack{n_{1} \geq 2, \ldots, n_{m} \geq 2 \\ n_{1} \cdots n_{m}=n}} a_{n_{1}}(F) \cdots a_{n_{m}}(F),
$$

where $\Omega(n)$ denotes the total number of prime factors of $n$. Thus the functions $F \mapsto b_{n}(F), n=2,3, \ldots$, are continuous on $\mathcal{S}^{\sharp}(1)$ with respect to $\varrho_{\mathcal{I}}$. In order to deal with the Euler product axiom, for $(n, m)=1$ we put

$$
g_{n, m}(F)=a_{n}(F) a_{m}(F)-a_{n m}(F),
$$

and for $\theta<1 / 2$ we write

$$
h_{n, \theta}(F)=n^{-\theta}\left|b_{n}(F)\right| ;
$$

note that $b_{n}(F) \ll n^{\theta}$ for some $\theta<1 / 2$ is equivalent to $\left|b_{n}(F)\right| \leq n^{\theta}$ for some $0<\theta<1 / 2$ and $n \geq n(\theta)$. Moreover, in order to deal with the Ramanujan conjecture axiom, for every $\varepsilon>0$ we define

$$
l_{n, \varepsilon}(F)=n^{-\varepsilon}\left|a_{n}(F)\right| .
$$

The three functions $g_{n, m}(F), h_{n, \theta}(F), l_{n, \varepsilon}(F)$ are continuous on $\mathcal{S}^{\sharp}(1)$ with respect to $\varrho_{\mathcal{I}}$, and $\mathcal{S}$ can be characterized as

$$
\begin{aligned}
\mathcal{S}= & \mathcal{S}^{\sharp}(1) \cap \bigcap_{(n, m)=1} g_{n, m}^{-1}(\{0\}) \cap \bigcup_{\substack{0<\theta<1 / 2 \\
\theta \in \mathbb{Q}}} \bigcup_{m=1}^{\infty} \bigcap_{n=m}^{\infty} h_{n, \theta}^{-1}([0,1]) \\
& \cap \bigcap_{\varepsilon \in \mathbb{Q}^{+}} \bigcup_{K=1}^{\infty} \bigcap_{n=1}^{\infty} l_{n, \varepsilon}^{-1}([0, K]),
\end{aligned}
$$

and the result follows as for $\mathcal{S}^{\sharp}$, thus proving the lemma.

Theorem 1 follows at once from (2.1) and Lemmas 2 and 3.

To prove Theorem 2, let $I \in \mathcal{I}, B \in \mathcal{B}(\mathcal{I})$ and $\mathcal{S}^{\sharp \sharp}(R)$ be as in $(2.1)$. Writing

$$
B_{R}=B \cap \mathcal{S}^{\sharp \sharp}(R),
$$


we see that $B_{R}$ is a Borel set of the compact metric space $\left(\mathcal{S}^{\sharp}(R), \varrho_{\mathcal{I}}\right)$. Moreover, by Proposition 4.2 of $[8],\left(\mathcal{S}^{\sharp}(R), \varrho \mathcal{I}\right)$ is a Polish space (see Definition 3.1 of [8]). Hence, by Theorem 13.7 (see also p. 85) of [8], $B_{R}$ is analytic (in Suslin's sense, see Definition 14.1 of [8]). Therefore, by Proposition 14.4 of $[8], I\left(B_{R}\right)$ is analytic as well since $I$ is continuous from $\left(\mathcal{S}^{\sharp \sharp}(R), \varrho \mathcal{I}\right)$ to $\mathbb{C}$ (and hence is a Borel map, see pp. $70-71$ of [8]) and $\mathbb{C}$ is obviously a Polish space. Finally, by Theorem 21.10 of $[8], I\left(B_{R}\right)$ is Lebesgue measurable, and hence

$$
I(B)=\bigcup_{R=2}^{\infty} I\left(B_{R}\right)
$$

is Lebesgue measurable as well.

The proof of Corollary 1 is very simple. Let $I$ be a continuous invariant and $\mathcal{I}=\left\{I_{0}, I\right\}$. Since $B \in \mathcal{B}\left(I_{0}\right)$, it follows that $B \in \mathcal{B}(\mathcal{I})$, hence $I(B)$ is Lebesgue measurable by Theorem 2 .

We need two lemmas for the proof of Theorem 3. We recall that a topological semigroup $(G, \cdot)$ is a semigroup where the multiplication - from $G \times G$ to $G$ is continuous.

LEMma 4. Let $\mathcal{I}$ be a countable family of continuous invariants and suppose that every $I \in \mathcal{I}$ is additive or multiplicative. Then $\left(\mathcal{S}^{\sharp \sharp}, \varrho_{\mathcal{I}}\right)$ is a topological semigroup.

Proof. We have to prove that the usual multiplication in $\mathcal{S}^{\sharp}$ is continuous with respect to the metric $\varrho_{\mathcal{I}}$. Let $I \in \mathcal{I}$ and write $*$ for the sum (resp. product) if $I$ is additive (resp. multiplicative). Let $F_{m} \rightarrow F_{0}$ and $G_{m} \rightarrow G_{0}$ be two convergent sequences in $\left(\mathcal{S}^{\sharp \sharp}, \varrho_{\mathcal{I}}\right)$. Since the functions in Lemma 1 are continuous, we see that as $m \rightarrow \infty$,

$$
I\left(F_{m} G_{m}\right)=I\left(F_{m}\right) * I\left(G_{m}\right) \rightarrow I\left(F_{0}\right) * I\left(G_{0}\right)=I\left(F_{0} G_{0}\right)
$$

for every $I \in \mathcal{I}$, and

$$
a_{n}\left(F_{m} G_{m}\right)=\sum_{d \mid n} a_{d}\left(F_{m}\right) a_{n / d}\left(G_{m}\right) \rightarrow \sum_{d \mid n} a_{d}\left(F_{0}\right) a_{n / d}\left(G_{0}\right)=a_{n}\left(F_{0} G_{0}\right)
$$

for every $n \in \mathbb{N}$. Hence $F_{m} G_{m} \rightarrow F_{0} G_{0}$ with respect to $\varrho_{\mathcal{I}}$, and the lemma follows.

Recalling that $B_{R}$ is defined by (2.6), we have

Lemma 5. Let $\mathcal{I}$ be a countable family of continuous invariants, let $B \in$ $\mathcal{B}(\mathcal{I})$ with $1 \in B$, and $G$ be the semigroup generated by $B$. Then

$$
G=\bigcup_{R=2}^{\infty} \bigcup_{k=1}^{\infty} B_{R}^{k} .
$$


Proof. The inclusion $\supset$ is obvious. To prove the opposite inclusion, given $F \in G$ we have $F(s)=\prod_{j=1}^{k} F_{j}(s)$ with some $k \in \mathbb{N}$ and $F_{j} \in B$. Then $F_{j} \in \mathcal{S}^{\sharp \sharp}\left(R_{j}\right)$ for some $R_{j}$, hence writing $R=\max \left\{R_{1}, \ldots, R_{k}\right\}$ we have $\left\{F_{1}, \ldots, F_{k}\right\} \subset B_{R}$. Therefore $F \in B_{R}^{k}$, and the lemma follows.

In order to prove Theorem 3, we first note that clearly $\{1\} \in \mathcal{B}(\mathcal{I})$, and we may always assume that $G$ is generated by a set $B \in \mathcal{B}(\mathcal{I})$, where $1 \in B$ (in fact, if $B$ is an $\mathcal{I}$-Borel set then $B \cup\{1\}$ is an $\mathcal{I}$-Borel set as well and generates the same semigroup). The proof of Theorem 3 now follows the lines of the proof of Theorem 2, hence we only give a sketch. $B_{R}$ is a Borel set of the Polish space $\left(\mathcal{S}_{\sharp \sharp}(R), \varrho_{\mathcal{I}}\right)$, hence it is analytic in Suslin's sense. Moreover, by Lemma 4, multiplication is a continuous function, therefore $B_{R}^{k}$ is also analytic. Since the invariant $I$ is continuous, $I\left(B_{R}^{k}\right)$ is analytic as well, and hence Lebesgue measurable. Thus, by Lemma $5, I(G)$ is Lebesgue measurable.

The proof of Corollary 2 is similar to the proof of Corollary 1.

Given a set $\mathcal{A} \subset \mathbb{R}, \mathcal{A}+\mathcal{A}$ denotes as usual the set of real numbers of the form $a+a^{\prime}$ with $a, a^{\prime} \in \mathcal{A}$. In order to prove the first part of Theorem 4 we recall that if $\mathcal{A}$ is measurable with $\mu(\mathcal{A})>0$, then $\mathcal{A}+\mathcal{A}$ contains an open interval; see Exercise 19 of Ch. 9 of Rudin [11]. Suppose now that $\mu(I(G))>0$. Since $G$ is a semigroup and $I$ is additive, we have

$$
I(G)+I(G) \subset I(G),
$$

hence there exists an interval $(a, b) \subset I(G)$. Therefore, again since $G$ is a semigroup, for every positive integer $k$ we have $(k a, k b) \subset I(G)$. Thus $I(G)$ contains arbitrarily long intervals. Let $F_{0} \in G$ with $I\left(F_{0}\right) \neq 0$ and let $U_{0} \subset I(G)$ be an interval of length $>\left|I\left(F_{0}\right)\right|$. Then

$$
\bigcup_{k=1}^{\infty}\left(k I\left(F_{0}\right)+U_{0}\right) \subset I(G),
$$

and such a union is a half-line, thus proving the first part of Theorem 4 .

The second option of the second part of Theorem 4 follows at once from the first part. In fact, let $I$ be multiplicative with values in $\mathbb{R}^{+}$. Write $\log I(G)=\{\log I(F): F \in G\}$. The function $F \mapsto \log I(F)$ is a real-valued additive continuous invariant. Moreover, if $\mu(I(G))>0$ then $\mu(\log I(G))>0$ as well, so $\log I(G)$ contains a half-line by the first part of Theorem 4 , and hence $I(G)$ contains a half-line too.

In order to prove the first option of the second part of Theorem 4, we first remark that a variant of the above mentioned exercise reads as follows. Let $\mathcal{A} \subset T^{1}$ and write $\mathcal{A} \mathcal{A}=\left\{a a^{\prime}: a, a^{\prime} \in \mathcal{A}\right\}$; if $\mathcal{A}$ is measurable and $\mu(\mathcal{A})>0$, then $\mathcal{A} \mathcal{A}$ contains an arc. Suppose now that $\mu(I(G))>0$ and 
argue as in the first part. Since $I$ is multiplicative we have

$$
I(G) I(G) \subset I(G),
$$

thus $I(G)$ contains an arc. Hence there exists $F_{0} \in G$ such that $I\left(F_{0}\right)=$ $e^{2 \pi i \theta_{0}}$ with $\theta_{0} \notin \mathbb{Q}$, therefore the set $\left\{I\left(F_{0}^{k}\right)\right\}_{k \in \mathbb{N}}$ is dense in $T^{1}$. But then

$$
T^{1}=\bigcup_{k=1}^{\infty} I\left(F_{0}^{k}\right) I(G) \subset I(G),
$$

and Theorem 4 is proved.

Acknowledgments. This research was partially supported by a MIUR grant Cofin2004, by the Foundation for Polish Science and by KBN grant 1 PO3A 00826.

\section{References}

[1] J. B. Conrey and A. Ghosh, On the Selberg class of Dirichlet series: small degrees, Duke Math. J. 72 (1993), 673-693.

[2] E. Hecke, Lectures on Dirichlet Series, Modular Functions and Quadratic Forms, Vandenhoeck \& Ruprecht, 1983.

[3] J. Kaczorowski, Axiomatic theory of L-functions: the Selberg class, in: Analytic Number Theory, C.I.M.E. Summer School (Cetraro, 2002), A. Perelli and C. Viola (eds.), Lecture Notes in Math. 1891, Springer, 2006, 133-209.

[4] J. Kaczorowski and A. Perelli, On the structure of the Selberg class, I: $0 \leq d \leq 1$, Acta Math. 182 (1999), 207-241.

[5] - - - The Selberg class: a survey, in: Number Theory in Progress, Proc. Conf. in Honor of A. Schinzel, ed. by K. Győry et al., de Gruyter, 1999, 953-992.

[6] - - - On the structure of the Selberg class, II: invariants and conjectures, J. Reine Angew. Math. 524 (2000), 73-96.

[7] - - -, On the structure of the Selberg class, IV: basic invariants, Acta Arith. 104 (2002), 97-116.

[8] A. S. Kechris, Classical Descriptive Set Theory, Grad. Texts in Math. 156, Springer, 1995.

[9] A. Perelli, A survey of the Selberg class of L-functions, part I, Milan J. Math. 73 (2005), 19-52.

[10] -, A survey of the Selberg class of L-functions, part II, Riv. Mat. Univ. Parma (7) 3* (2004), 83-118.

[11] W. Rudin, Real and Complex Analysis, 3rd ed., McGraw-Hill, 1987.

Faculty of Mathematics and Computer Science

A. Mickiewicz University

Umultowska 87

61-614 Poznań, Poland

E-mail: kjerzy@amu.edu.pl
Dipartimento di Matematica

Università di Genova

via Dodecaneso 35

16146 Genova, Italy

E-mail: perelli@dima.unige.it

Received on 14.11.2006

and in revised form on 24.7.2008 